\title{
CALL: The Need for Evaluation Paradigms
}

\author{
Dr. Abdellatif Elshazli Y. Ali
}

Faculty of Education, Benha University

\section{Abstract}

7 he evaluation of CALL from the perspective of gross comparisons of CALL versus classroom learning outcomes is an approach unlikely to shed light on the problem or solution of instructed SLA. It creates an irony wherein the most sophisticated modern tool is investigated through the most crude and outdated educational research methods (Chapell 2005)This paper reviews current methods of evaluation of CALL as an instructional solution to the problem of instructed SLA, on the basis of theory of conditions (cognitive and socio-affective) that ideally should be created for optimal/successful learning environments and effective task-based instruction.Evaluation of CALL task cannot be a categorical decision about effectiveness. Instead, it results in an argument indicating in what ways a particular task is appropriate for particular learners at a given time, i.e., CALL task appropriateness. CALL task evaluation denotes three levels of analysis: CALL software, teacher-planned activity, and learners' performance during activities. Evaluation argument is constructed on the basis of both judgemental and empirical analyses. The former examines characteristics of the software and t/task while the latter addresses the details of CALL taskuse and learning outcomes in terms of principles /criteria drawn from theory and research on conditions for instructed SLA. The criteria for CALL appropriateness are applied in view of the stated purpose of a CALL task at various stages of instruction. Examples of CALL task evaluation, a judgemental as well as an empirical research tasks/activities, are provided.

\section{Introduction}

In the $21^{\text {st }}$ century, the use of computer technology for language teaching and learning has become a fact of life with important implications for all those concerned with facets of instructed Second Language Acquisition (SLA). They need to grasp the nature of unique technology-mediated tasks that learners can engage in for language acquisition. Language learners typically use computers for variety of purposes. One 
challenge for language teachers is to shape some of the computer-using experiences into language learning experiences.

To meet the challenge, the study of the features of computer-based tasks that promote learning should be a concern for teachers as well as researchers who wish to contribute to/enrich knowledge about instructed SLA.

Two trends have caught the imagination of language teachers in recent years. One is the growing emphasis on communication. Theoreticians and practitioners are stressing 'communicative competence" as the goal for language teachingi.e., on using language for purposes beyond merely getting the right answer and pleasing the teacher. Communicative teaching concentrates on genuine exchange of information/appropriate message in a given social context, i.e. communicative activities and meaningful practice. The second trend is computer-assisted instruction (CAI). Over the last three decades, CAI has witnessed explosive growth. Rapid developments in computer technologies are obviously a driving force, but so too, is the increasing number of computer-literate language teachers. How can these two trends converge to the benefit of second or foreign language teaching and learning/education?

The question is not one of evaluating what is out there but of deciding 'what ought to be done.' To do so, language teachers/teacher trainers/educators have to look at a lot more than just computers. They have to consider what foreign language learning and teaching are all about: what works, what doesn't, and why. They need to reflect more on language learning process and ask some hard questions: Does the computer help teachers accomplish what they are trying to do? Does the program actually do anything related to the goals they set for students? Or how do computer programs make a difference in language teaching/learning ? (Underwood ,1984).

The answer to such a question requires a survey of the potentials of computer applications - programs intended for direct use by students - as a solution to the problem of instructed 
SLA. The purpose of this paper is to offer a principled basis for deciding "what ought to be done" in terms of principled design of CALL tasks. It will examine what is being and could be done with computers in language teaching and see how well it fit, or doesn't fit, the premises for communicative CALL, principles, and criteria for evaluation of computer applications in instructed SLA.

Computer Applications in Second Language Acquisition (CASLA) or CALL began within the field of educational technology and was therefore shaped by perspectives in education as well as by computer sciences. Computer-based learning activities, called 'courseware', were developed using general-purpose programing languages to implement the instructional design for CALL. Used as an adjunct to classes in ESL, courseware was seen as a supplement to rather than a replacement for classroom instruction.

The more sophisticated microcomputers called for more sophisticated programing in the form of authoring tools such as Intelligent Tutoring Systems (ITS) for language learning, Intelligent CALL. The philosophy behind the ITS entails that instruction should be designed to explicitly focus on learners' linguistic needs. The widespread use of networked computers expanded the characteristics of CALL activities. Instructional courseware/activities were developed around the computerlearner interactions in which the language-learning objectives of CALL program as well as the learning path(s) were predetermined/precisely specified. Collaborative/grouporiented activities were built around learner-learner interaction through networked computers where they work with, learn with, or draw on programs pedagogical /linguistic resources. With the development of the Local Area Network or the Internet, computer-mediated communication was put into pedagogical use for teaching in and across second language classrooms, i.e., facilitative/communicative activities. The facilitative software serves as a tool in language learning activities, i.e., to minimize the inauthentic labor involved in the learning process. This computer-assisted discussion provided a written record of 
learners' on-line/electronic/ interpersonal communication which could be examined from the perspective of discourse analysis and SLA.

Internet technology expanded possibilities for data collection in SLA research as well. Researchers took advantage of such technology to design computer-assisted experiments and to implement learning conditions such as explicit/deductive or implicit/inductive learning of simple grammar rules/examples. Innovative computer-delivered tasks were devised for gathering data about interlanguage knowledge and processing strategies learners use while performing in classroom activities.

Instructional network /computer lab introduced universal access to materials/ information as well as hybrid applications offering learners the best of both worlds such as discussion groups, e-mail lists, web-based language learning. From the learners' perspective, interesting opportunities for autonomous language learning and self-assessment became widely available. CALL activities included computer-mediated communication with learners in class and/or in other parts of the world, as/synchronously. Teachers' interest in Internet activities/resources suggests a new internet pedagogy conceptualized/build on social/computing and collaborative L2 teaching and learning (Warschauer, 2000).

The idea of acquisition through manipulation of a responsive environment attracted CALL developers seeking ways in which the computer could create/provide good contexts for implicit acquisition. CALL activities create a 'discovery-rich environment' for the student to explore and interact with' through combination of video and NLP techniques/systems which could analyze/process and respond in real time to written input, i.e., technology-enhanced language learning.

The primary impact of SLA theory was contributed by Krashen's (1982) ideas that CALL could be claimed to promote acquisition rather than learning. The computer can serve not only the conscious process of explicit learning but can also 
facilitate acquisition. Developing 'communicative CALL', consistent with Krashen's prescriptions for creating a good context for acquisition, involved the use of artificial intelligence techniques (NLP programs such as games and collaborative learning activities) to recognize learners' input to the computer and to generate responses, a meaningful conversation between computer and learner. Evaluation of CALL tended to be the developers' or users' opinion about the extent to which an activity seemed communicative on the basis of type of tasks learners engage in. Communicative (learner-controlled) tasks such as text reconstruction and computer-assisted concordance activity were argued to empower learners to investigate vocabulary use and grammatical collocation on their owncustomized CALL materials. Another influence of SLA theory came from research on the role of individual difference on the effectiveness of different instructional approaches and the desirability of CALL, i.e., investigating learning styles/learner strategies and task variables in CALL.

Conventional CALL evaluation procedures employing group mean differences are inappropriate for examining CALL from perspectives consistent with SLA classroom research. Comparative research (control-comparison group design) attempting to illustrate the superiority of computers over some other medium/ mode for language instruction should forever be abandoned' (Pederson, 1994)...because the essential variablescomputer capabilities/coding options, learner differences, learning task-that might make a difference in a given languagelearning context cannot be isolated, manipulated, and examined adequately. These studies, though provide little usable information outside of their specific setting, in one way or another, fall into the trap of attempting to attribute learning gains to the medium itself rather than to the way the medium was manipulated to affect achievement. In addition, comparative research on computer-assisted instruction versus non-CAI is incapable of providing generalizable results for theoretical limitations. First, there is no way to replicate the conditions of the experiment exactly, therefore, results lack 
consistency/reliability from one place to another. Second, there is no valid way to ascribe with confidence the causes for differences in independent variables to the independent ones, without adequate controls of all possible causes for learning differences. Third, such studies usually fail to hold hypotheses based on language learning theory, and, as a result, to integrate results into theoretical research base, and to utilize outcomes for adjustments in classroom teaching that will improve second language learning. CALL, in and of itself, does not result in more and better learning, it is the specific way instruction is coded in CALL software that has the potential of affecting learning positively, for specific learners in specific contexts.

The bulk of research on computers and learning in educational environments has focused on the cognitive aspects of learning. Yet, theory and research in SLA classrooms indicate that social interactional environments of classroom are also crucial factors that affect language learning in important ways. Research concerns evolved away from the idea that solely the input that learners receive through communicative activities would promote acquisition. Learners need to 'notice and interact' with the linguistic input in order to acquire the target language. That is, using computer-assisted experimental materials that operationalize theoretically different learning conditions in order to direct learners' attention. Researchers advocated study of CALL within its larger classroom and sociocultural context consistent with theory and research in SLA, i.e., social interactional environments of the classroom.

Developments in some current philosophies and practices of Computer Applications in SLA were precipitated by work in related fields/academic areas of education, linguistics, and psychology. Six computer-related sub-disciplines have made significant contributions to CASLA: educational technology, computer-supported collaborative learning, computational linguistics, and corpus linguistics, artificial intelligent and computer-supported assessment. However, the specifics of evaluation for Second Language Learning must be developed 
from the relevant perspectives in applied linguistics (Chapelle 2005). Review of the first two sub-disciplines is of particular relevance to scope/context of this paper.

Computer-Assisted Language Learning (CALL) has its roots in educational technology, a specialization within the study of education. The general goal /concern /primary question, in educational technology, is: How can computers best be used to improve learning? Researchers and practitioners attempt to devise the best ways of using computer technology for instruction across subject areas and to design valid ways of evaluating its effectiveness. Both computer-assisted instruction and evaluation methods were strongly influenced by the philosophy and practice of the 'system approach' to instructional design, which has an empiricist orientation to leaning and instruction. That is, learning is defined by breaking/analyzing it up to components as observable behaviors and designing instruction accordingly. Perspectives from educational technology on how to evaluate the learning activities view research as adopting experimental or quasi-experimental design and product-oriented. The debate on ideal research design/paradigm for investigating educational technology continues today.

Despite the apparent independence of CALL as a professional community, its practices and philosophies have remained closely tied to those of educational technology, i.e., the systems approach to courseware design. Influenced by this thinking, early CALL evaluation research attempted to demonstrate its effectiveness, using quasi-experimental research designs, comparing cognitive and effective outcomes of learners who participated in Computer-Based Instruction with those in traditional/regular classrooms. In some studies, CALL users performed better than learners who did not use CALL, no differences were found, or control group performed better. Results of this research do not warrant conclusions to be drawn about the 'computer as a teaching method' (Chapelle 2005). 
Problems with equating the computer to teaching method have been noted by CALL researchers, but the need for evaluation paradigms to rely on a more complex view of learning has been articulated most clearly by educational technology. Questioning the meaningfulness of summary statements making claims about the overall effects of the computer on learning, Clark (1994) explains that 'instructional methods have been confounded with instructional media and that it is the methods which influence learning'. 'Method' is defined as the 'structural' characteristics of the tasks for learners which engender the processes and strategies necessary for learning. In contrast to 'methods', 'media' refer to a means of delivering methods/instruction to learners. The argument is that any 'method' produced in a media-assisted format can also be delivered by other means and therefore, media may influence the cost or speed (efficiency) of learning, but methods are causal in leaning. What is needed rather than studies focused on the computer are studies investigating relevant task variables in computer-assisted environments. A second problem of studies investigating computer effects is that they tend to ignore characteristics of individual learners. These problems of task and learner definition do not question the basic tenets of treatmentoutcomes experimental design in educational research; they suggest instead that the paradigm has been poorly implemented.

A paradigm is defined as 'a researcher's specific stance on how knowledge can be revealed/uncovered' (Egbert and Sanden, 2014,32 ). A research paradigm is influenced by researcher's conceptual framework (worldview) and epistemology (whether there is Truth or truths). A researcher's paradigm reflect in the way research is designed, how data is both collected and analyzed, and how research results are presented. It allows researchers to identify their role in research process, determine course of research, and distinguish other perspectives. In addition, explicit mention and recognition of the researcher's stance allows consumers/readers of research to better understand and evaluate research results, conclusions, and biases. 
Four general categories of paradigms are recognized in academic literature. Positivist researchers believe that knowledge is based on observed experience and can be obtained through experiment. Truth is objective, measurable and generalizable. Positivism underscores systematic 'scientific method' that emphasizes logic and empirical results and works toward verification of hypothesis. Interpretivist paradigms hold that objectivity is useful but not necessarily attainable ideal, particularly in natural settings/classrooms. Post-positivists underscore that reality is multilayered, complex, and can have multiple interpretations. Researchers emphasize human interaction with phenomena and suggest a mix of qualitative and quantitative methodological frameworks. Critical scholars believe that truth can be revealed by exploring existing political conditions, contradictions, hidden agendas and benefits of current social order. They stress building awareness of multiple realities and allowing diverse voices to come to the fore. Constructivist paradigm emphasizes that reality is viewed and interpreted by the individual and group. Knowledge is subjective, contextualized, personally experienced, and that language and prior knowledge mediate individual realities. Findings are literally created through interaction between the researcher and people being researched. However, these categories of paradigms are not discrete/immutable entities, but may usefully overlap and can yield fruitful results (Egbert and Sanden 2014, 33-5).

The need for research to examine the processes occurring within the classroom culture rather than the effects of a single technology has been asserted by researchers in educational technology. Criticizing the outcome-oriented research methods associated with logical positivism, they argue that studies focusing on quantified outcomes of a group of learners fail to document the many contextual factors influencing the processes of learning within a classroom culture. Proponents of the social constructivist paradigm argue that investigating contexts of computer use supports the use of qualitative research methods. 
In short, some evaluation perspectives that are relevant to CALL have originated in the field of educational technology.

The area of 'computer-supported collaborative learning' (CSCL) is a branch of educational technology, but is motivated by distinct philosophies and practices. The general goal/primary concern/question is: How can computer-assisted activities be designed to promote learning through collaboration? Such paradigm shift in research, based on assumptions about the nature of learning and research practices, approaches learning and instruction as psychological matters, behavioristically or cognitively researchable by experimentation. The emerging paradigm 'cultural constructivist approach' encompasses a complex of philosophies and beliefs about the way that learning and experience are internalized and transferred. Constructivists argue that students' learning experience/context is critical to what they learn and how they are able to use it. The cultural dimension includes the essential role the social environment/context plays in learning, referred to as 'situated leaning' (Pritchard, 2014).

The constructivist perspectives most closely associated with CSCL have its roots in Vygotskyan cultural psychology (Lantolf and Appel 1994) which makes sense of "learning" by reference to the social structure of activity, rather than to the mental structure of the individual. It can include the computer software with which the learner interacts, in addition to other learners who collaborate in the same room or from remote locations through networked computers. Cultural constructivists hypothesize that the experience crucial for individual cognitive development takes place through interaction with others, and therefore key evidence for the quality of a learning activity should be found in the discourse that occurs in the collaborative environment. Therefore, Research on computer-assisted collaboration takes the form of qualitative content analysis of collaborative discourse. Some methodological approaches of CSCL, which overlap with those of SL classroom research, have been applied in collaborative CALL or internet activities (Warschauer, 2000). 
Developments in CALL benefit from authoring software, instructional practices, and methods of evaluation borrowed from these two areas. The corresponding concern in CALL is: How can collaborative computer-assisted language learning activities be designed to promote development of communicative L2 ability? Unique issues arise in teaching and evaluating the success of L2 learning. For example, application of systems approach from edtech to CALL can result in materials which break up language into component parts to teach in a hierarchical fashion - a practice runs contrary to accepted meaning-based approaches. Because of the unique /particular difficulties inherent in computer processing of natural language, design and evaluation of CALL through direct analogies with educational technology have not been successful. Cultural constructivist perspective of CSCL may solve the problem with development and evaluation of learning through collaboration/the use of language. However, collaboration, as used in many settings, is not targeted toward language learning. What is needed are theoretical and empirical based criteria for choosing among potential design options and methods for evaluating their effectiveness for promoting learners' communicative L2 ability.

Clark (1994) described the reason for lack of substantive progress in educational technology as 'we (students and faculty) tend to begin with educational and instructional solutions (an enthusiasm for some medium) and search for problems (a visible context) to establish evidence for our solution. If we begin by im/explicitly attempting to validate a belief about solutions to largely unexamined problems, we are less open to evidence that our intuitions might be very far off the mark'. This situation presents a problem for developing methodologies for CALL evaluations.

\section{The problem of instructed SLA:}

The theory of instructed second language acquisition attempts to explain how instructed learners acquire the kind of linguistic and practical knowledge needed to produce correct 
and appropriate sentences in a second language, i.e., develop language proficiency. To account for 'who learns how much of what language under what conditions', Spolsky (1989) proposes an interactive model consisting of 74conditions' governing different aspects of L2 learning. The theory examines the relationship between the kind of learning opportunities provided in classroom context and the learning outcomes that are likely to result in (Ellis 2009).

In a theory of instructed SLA, input is the raw data with which L2 learners work. The characteristics of the input in the classroom setting depend on the type of instruction. In instruction directed at teaching the linguistic code, L2 features are made artificially frequent in the input for some time resulting in an overuse of forms. The frequent and salient forms are more noticed and, therefore, potentially more learnable. Furthermore, teachers' treatment of learner errors may increase the salience of selected features, thus enhancing their noticeability and learnability. Code-oriented instruction may also provide learner with explicit information about the nature of linguistic features in the form of rules. Instruction aimed at providing learners with opportunities to communicate naturally (communicative activities) typically makes no attempt to manipulate the input or output in order to focus on specific items/features. The frequency of different items, therefore, is not predetermined but rather is the product of the language participants choose to use to complete a task. The shaping of input through modifications of teacher's talk involves changes of linguistic form in terms of simplification, elaboration, and redundancy. Comprehensible input results from input and, in particular, interactional modifications facilitates natural development of a second language. The input and interactional modifications helps to make input comprehensible and specific linguistic features more noticeable are important for acquisition.

Evaluation of CALL as a solution to the problem of instructed second language acquisition need to begin with an understanding of, or at least hypotheses about, the conditions 
that ideally should be created for ISLA. Research on instructed SLA addresses the issues of conditions of instruction and of communication tasks, i.e, investigates how specifics of the environment influence SLA. Research findings have great importance for educators: SL material writers, curriculum developers and classroom teachers. Egbert (1999) proposes conditions for optimal learning environments, based on Spolsky's (1989) summary of conditions for successful SLA-a theory of conditions in both cognitive and socio-affective domains. Skehan (1998) offers five guidelines, gleaned from current research base, for implementing effective task-based instruction: range of target structures, the utility condition, balanced goal development, focus on form through attentional manipulation, and cycles of accountability.

Range of target structures. SLA research findings indicate that learners will acquire particular structures or develop formmeaning connection when they are ready to. Instruction can help speed up the process by selecting a range of target structures for learning materials rather than keeping learners to a strict schedule of items to be acquired. That is learners need to be exposed to language within their grasp, not far beyond or beneath their abilities or needs.

Utility condition. It refers to the degree(s) of likelihood that a particular structure will be used by learners as they perform a task, as learning utility, for language learning. The utility criterion for task selection puts the teacher in the position of creating condition in which target structures might be practiced/used, rather than assuming teacher selects each structure one by one.

Balanced goal development. Skehan (1998) Three dimensions of language performance are associated with L2 tasks: fluency, accuracy, and complexity. Therefore, pedagogical tasks should be chosen to help learners develop in these ways. The balancing of development among these areas should occur through teachers' choice of tasks that alternatively provide opportunities for development of each. 
Focus on form through attentional manipulation. SLA Research findings indicate the learners need to 'notice and attend to' linguistic form for acquisition during meaning-based tasks. Focus on form often consists of a shift of attention to linguistic code features-by the teacher and/or one or more students-triggered by perceived problems with comprehension or production (Long and Robinson, 1998). Attention to form can also occur when learners modify their linguistic output because of problems in getting a message across/communication tasks. In addition to negotiation of meaning and modification of output, Skehan (1998) identifies six task characteristics which may help to manipulate attention/direct learners' attention to linguistic form in learning tasks: time pressure, modality, support, surprise, control, and stakes.

Cycles of accountability. It refers to learners' responsibility to keep track of what they are learning to and plan for their own development. Teachers therefore have the responsibility of drawing learners' attention to the need to be aware of the language they are acquiring, a cyclic/ongoing process as learners work with a variety of tasks.

Socio-affective conditions synthesize a construct of 'willingness to communicate' /use an L2 (WTC) as a situationspecific variable representing an intention to communicate at a specific time to a specific person. WTC is comprised of layers of underlying learners' predispositions.

Other factors include individual difference, learning situation, and available resources. These conditions for instructed SLA offer the most solid basis for making decisions about task selection. That is, they must be taken into account to inform the particulars of the evaluation criteria for CALL.

\section{Principles for CALL evaluation:}

Learners use computers for different purposes and therefore teachers, researchers and software developers are concerned about what kinds of CALL tasks may be beneficial. Given the need to make judgements about CALL, guidelines, checklists, and evaluation rubrics for CALL materials have been 
developed as a means of setting some criteria for what can be considered good CALL. Five principles for evaluating CALL are summarized as follows:

1. Evaluation of CALL is a context/situation-specific argument.

2. CALL should be evaluated through two perspectives: judgemental analysis of software and Teacher planned tasks and empirical analysis of learners' performance.

3. Criteria for CALL task quality should come from theory and research on ISLA

4. Criteria should be applied in view of the purpose of the task.

5. Language learning potential should be the central criteria in CALL evaluation.

Due to the complexity of factors to be considered in designing appropriate pedagogical L2 tasks, evaluation cannot be a categorical decision about effectiveness. Instead, an evaluation has to result in an argument indicating in what ways a particular CALL task is appropriate for particular learners at a given time. That is CALL task appropriateness needs to be evaluated on bases of evidence and rationales pertaining to task use in particular setting.

CALL evaluation can denote thdifferent levels of analysis. Judgemental analysis examines characteristics of the software/important features such as learner's control, interactivity, feedback, record keeping; and teacher's planned activity/task, the way in which teacher introduces and structures CALL activity, control or lack of control, what teachers do with the program. The third level of analysis focuses on empirical data reflecting learners' use of CALL and learning outcomesinteraction and negotiation of meaning. These two methods provide different and complementary information relevant to CALL evaluation.

\section{Criteria for evaluating CALL:}

Drawing from theory and research on conditions for ISLA, six criteria for CALL task appropriateness can be outlined as follows: 
1. Language leaning potential: the degree of opportunity present for beneficial focus on form, rather than simply an opportunity for language use.

2. Learner fit: the amount of opportunity for engagement with language under appropriate conditions given learner characteristics.

3. Meaning focus: the extent to which learners' attention is directed toward the meaning of language.

4. Authenticity: the degree of correspondence between CALL activity and target language activities of interest to learners out of classroom.

5. Positive impact: positive effects of CALL activity on those who participated in.

6. Practicality: the adequacy of resources to support the use of the CALL activity.

These criteria for CALL appropriateness need to be applied in view of the stated purpose of a task, such as performance goals for tasks including learners' fluency, accuracy, and complexity.

The criteria are intended to guide both judgemental and empirical analysis of CALL tasks. The judgemental analysis is intended to assess the appropriateness of a task for particular learners at a particular point in time, i.e., relative to the setting in which they are used. A set of questions can be used to examine/focus on both aspects of individual task defined by software and those designed by the teacher as follows:

1. Lang learning potential: Do task conditions present sufficient opportunity for beneficial focus on form?

2. Lang fit: Is the difficulty level of targeted linguistic forms appropriate for learners to increase their language ability? Is the task appropriate for learners with characteristics of the intended learners?

3. Meaning focus: Is learners' attention directed primarily toward meaning of the message?

4. Authenticity: IF/Is there a strong correspondence..., Will learners be able to see the connection between the CALL tasks and tasks outside the classroom? 
5. Impact: Will learners learn more about target language and about strategies for language leaning through the use of the task? Will instructors observe second language pedagogical practices by using the task? Will both learners and teachers have positive learning experience with technology through use of the task?

6. Practicality: Are hardware, software, and personnel resources sufficient to allow CALL task to succeed?

Logical analysis of CALL activity requires description of the complete task and the contest in which it is used, i.e. contextspecific evaluation. However, judgemental analysis is only one part of overall task evaluation. Empirical analysis is needed to provide evidence of the extent to which the judgemental analysis accurately reflects how learners work with the CALL task.

Judgemental analysis offers a methodology for making systematic hypotheses about the benefits to be attained through CALL tasks. As such, hypotheses stand in need of support through empirical data, because as SLA research has shown, 'students are often doing something different from what teachers assume/plan they are doing. That is, it is necessary to identify observable data that provide evidence of CALL qualities.

Limitations of the study of learning outcomes has been well researched in literature on educational technology as well as in CALL. Empirical research methods for evaluating L2 classroom instruction tasks have given up solely measurement of learning outcomes in favor of investigating classroom/learning processes (Johnson 1995). A research result showing language learning outcomes that can be attributed to particular features of instruction is seductive and cause for suspicion. Examination of the qualities outlined above might best be studied by examining learning outcomes related to particular task features, i.e. language learning potential, whereas the study of learning outcomes would offer little or nothing to questions about task authenticity. In short, each of the qualities implies particular research questions and associated methods. 
When applied to CALL, the research methods suggested above are implemented somewhat differently, largely because the computer is able to record the language and non-linguistic moves/behavior that the learner makes than other forms of observation. Computer-gathered data prove useful for investigating some questions about appropriateness. But the issue is what kind of evidence is required to address a particular research question, i.e., what we should do with the data/information. Research questions about CALL need to be developed in view of the qualities about which evidence is sought. The following is an outline of general research questions that would address each of the CALL qualities.

1. Lang learning potential: what evidence suggests that learner has acquired the target forms that were focused on during CALL task? What evidence indicates that learners focused on form during CALL task?

2. Learner fit: what evidence suggests that targeted linguistic forms are at an appropriate level of difficulty for learners? What evidence suggests that task is appropriate to learners' individual characteristics, e.g., age, learning style, computer experience?

3. Meaning focus: what evidence suggests that learners' construction of meaning aids language learning? What evidence indicates that learners use language during task for constructing and interpreting meaning?

4. Authenticity: what evidence suggests that learners' performance in CALL task correspond to what one would expect to see outside CALL task? What evidence suggests that learners see connection between CALL task and tasks outside classroom?

5. Impact: what evidence suggests that learners learn more about target language and strategies for language learning through use of the task? What evidence suggests that instructors engage in sound second language pedagogical practices by using task? What evidence suggests that 
learners and teachers had a positive experience with technology through use of the task?

6. Practicality: what evidence suggests that hardware, software, and personnel resources prove to be sufficient to allow task to succeed?

Empirical research demonstrating the language learning potential of a CALL task need to show that learners have improved their control of particular aspects of target language focused on in the activity/L2 input, i.e. CALL tasks with or without condition hypothesized to be beneficial. To the extent that the conditions are carefully defined in such tasks, results can contribute to principles for designing CALL tasks with language learning potential.

\section{Computer-assisted SLA Research task :}

is defined as one in which learners are expected to work on target language interactively with computer program or with other people through the medium of a computer-as a regular part of instruction, testing, or explicitly introduced as research tasks. One type of CASLR is to gather evidence about effects of instructional conditions of learning, by operationalizing each condition through CALL. A second type of CASLR is used for assessment, i.e., to make inferences about aspects of the learners' language ability and learning. In the former case, learners work on a task while the computer collects data on their performance; in the latter case, the researcher may also gather data such as learner' introspective comments.

Investigating instructional conditions. The computer is used to control the instructional conditions/design features/treatments and document developing knowledge. Robinson's (1996) lab-like experiment comprised four conditions of instruction constructed through the methods/types of presentation to the learners in each of four groups. Other conditions/research pertinent to theory of SLA, and applied studies investigating CALL instructional tasks or CALL materials include computer games and e-dictionary use. 
Evaluation of learning condition. Computer-Assisted SL research tasks used for implementing instructional conditions need to be interpreted and evaluated. The set of criteria for CALL research tasks/CASLR in classroom research include: quality of operationalization of learning condition, participation of learners in condition, and the generalizability of results, as follows:

1. Operationalization of learning condition: the degree to which the theoretically defined learning condition is produced in the CASLR task and the degree to which subjects participate in the learning condition, available during instruction.

2. Generalizability: the degree of correspondence between the learning condition in the experimental CASLR task and the learning condition/context outside the research setting to which results are to generalize.

Operationalization of Learning Condition. The concern for operationalization is that researcher has correctly constructed the operational conditions theorized to produce particular effects (logical analysis) and that learners actually participate as intended in those conditions (empirical analysis) of CALL tasks. The need for a detailed explanation of conditions of instruction/micro-level design features incorporated into computer application, such as presentation of incidental condition or provision of opportunities for interactional modification... computer-based materials offer a different range of options for investigating features of instructional design. Therefore, it is necessary to justify rationally the psycho-and socio-linguistic conditions believed to be operationalized by particular design features

At the other level, the quality of a learning condition depends on the extent to which learners took part/engage in the condition available during instruction. Experimental treatment be ended with retrospective interview or questionnaire/data to assess the extent to which learners participate. Another means of producing relevant evidence for conditions is through computer- 
documented data indicating the processes through which learners complete à task, i.e., actual working processes of learners.

Generalizability. External or ecological validity refers to the extent to which results can be considered relevant to contexts beyond the research/experimental settings. A primary concern of ISLA researchers should be establishing the scope over which research results of a study can be generalized. Evaluation of generalizability therefore relies on the authenticity of the research task relative to other task to which generalizations might be made. CASLR investigating specific conditions for ISLA, particularly about explicit versus implicit learning conditions in L2 classrooms.

\section{References:}

Ali, Hossam. 2016. The effectiveness of using the Corpus Approach in developing writing skills and reducing writing anxiety among EFL student teachers. Unpublished Ph.D. thesis. Faculty of Education, Benha University.

Al-Sayed, Rania. (2016). The effectiveness of using "WebQuest" approach in developing EFL writing strategies and creative writing skills among experiments preparatory school pupils. Unpublished Ph.D. thesis. FOE, Benha University.

Chapelle, Carol A. 2005. Computer Applications in Second Language Acquisition: Foundations for teaching, testing and research. Cambridge: CUP.

Egbert, joy \& Sanden, Sherry. 2014. Foundations of Educational Research: Understanding theoretical components. New York: Routledge.

Ellis, Rod. 2009. SLA Research and Language Teaching. Oxford: OUP.

Pritchard, Alan. 2014. Ways of Learning: Learning theories and learning styles in the classroom. 3rd ed. London: Routledge. Pusak, James. 1984. Problems and prospects in foreign language computing. In Smith, Wm. (ed). 1994. Modern Media in Foreign Language Education: Theory and implementation. Chicago, Illinois: National textbook company. 
Sallam, Ahmed. 2013. The effectiveness of a corpus-centered program in developing lexicogrammatical materials and linguistic competence among EFL prospective teachers. Unpublished Ph.D. Dissertation. FOE, Damanhour University.

Shaban, Reda. (2010). The effectiveness of a suggested computer-based program on developing some EFL oral communication skills among prep stage students. Unpublished M.A. thesis. FOE, Benha University.

Underwood, John. 1984. Linguistics, computers, and the language teacher: A communicative approach. Rowley, Mass.: Newbury House Publishers, Inc.

Warschauer, Mark; Shetzer, Heidi; and Meloni, Christine. 2000. Internet for English Teaching. Alexandria, Virginia: TESO, Inc.

Wyatt, David. 1984. Applying pedagogical principles to CALL courseware development. In Smith, Wm. (ed). 1994. Modern Media in Foreign Language Education: Theory and implementation. Chicago, Illinois: National textbook company. 
